\title{
A real-world evaluation of indacaterol and other bronchodilators in COPD: the INFLOW study
}

\author{
This article was published in the following Dove Press journal: \\ International Journal of COPD \\ 5 October 2015 \\ Number of times this article has been viewed
}

\section{Georges Juvelekian' \\ Waleed El-Sorougi ${ }^{2}$ \\ Chaicharn Pothirat ${ }^{3}$ \\ Faisal Yunus ${ }^{4}$ \\ Teresita De Guia ${ }^{5}$ \\ Han-Pin Kuo ${ }^{6}$ \\ Shalma Basu Patnaik ${ }^{7}$ \\ Virginia Pilipovic ${ }^{8}$}

'St Georges Hospital University Medical Center, Ashrafieh, Beirut, Lebanon; ${ }^{2}$ Chest Department, Faculty of Medicine, Helwan University, Cairo, Egypt; ${ }^{3}$ Division of Pulmonary and Critical Care, Faculty of Medicine, Chiang Mai University, Chiang Mai, Thailand; ${ }^{4}$ Department of Pulmonology and Respiratory Medicine, Faculty of Medicine,

University of Indonesia, Persahabatan Hospital, Jakarta Timur, Indonesia;

${ }^{5}$ Department of Pulmonary Medicine, Philippine Heart Center, Quezon City, Philippines; 'Linkou Chang Gung Memorial Hospital, Taoyuan Hsien, Taiwan, ROC; ${ }^{7}$ Novartis Healthcare Pvt Ltd, Rangareddy, Telangana, India; ${ }^{8}$ Novartis Pharma AG, Basel, Switzerland
Correspondence: Georges Juvelekian St Georges Hospital University Medical Center, Post Box 166378, Ashrafieh, Beirut I I002807, Lebanon

Tel +96I 3497574

Fax +96| I 582560

Email juveleg@hotmail.com

\begin{abstract}
Aim: INFLOW (INdacaterol eFfectiveness and utiLizatiOn in COPD: real World evaluation) was a prospective, noninterventional study assessing the effectiveness and safety of long-acting bronchodilators in patients with chronic obstructive pulmonary disease (COPD) from the Middle East, Asia, and South Africa.

Methods: Patients newly prescribed or switched to indacaterol or other long-acting $\beta_{2}$-agonist (LABA), or tiotropium (monotherapy or in combination) were evaluated over 6 months. The primary endpoint was the clinical COPD questionnaire overall score at the end of the study.

Results: Data were analyzed from 1,710 patients (mean postbronchodilator forced expiratory volume in 1 second, $59 \%$ predicted) who received indacaterol $(n=1,179)$, other LABA $(n=68)$, tiotropium $(n=271)$, indacaterol plus tiotropium $(n=167)$, or other LABA plus tiotropium $(\mathrm{n}=25)$. Across treatments, clinical COPD questionnaire overall score improved from baseline by $0.81-1.26$ points (all $P<0.0001$ ), $63 \%-84 \%$ of patients were satisfied/very satisfied, and physicians rated effectiveness as good/very good in $63 \%-80 \%$ of cases. The indacaterol inhaler was rated easy/very easy to use by the majority of patients, and physicians considered its use clearly understood by most patients. All treatments had acceptable tolerability.

Conclusion: In real life clinical practice across a diverse region, indacaterol and other long-acting bronchodilators improved health status and were well regarded by patients and physicians.
\end{abstract}

Keywords: long-acting bronchodilator, indacaterol, chronic obstructive pulmonary disease, noninterventional study, Middle East, Asia

\section{Introduction}

Chronic obstructive pulmonary disease (COPD) is a common, preventable, and treatable disease and a leading cause of morbidity and mortality worldwide. ${ }^{1,2}$ COPD prevalence varies in different regions. ${ }^{3}$ A large epidemiological study of $>60,000$ interviewees from 11 countries of the Middle East, North Africa, and Pakistan (BREATHE study) ${ }^{4}$ reported an overall prevalence of $3.6 \%$, while the Asia-Pacific regional working group reported an overall prevalence of $6.3 \%$ across 12 Asian countries. ${ }^{5}$ Prevalence may differ not only between but also within countries. ${ }^{6}$ The prevalence of COPD in India is unknown, but chronic bronchitis has been estimated to affect $6.5 \%-7.7 \%$ of the rural population. ${ }^{7}$ The BREATHE study also reported a high burden of disease, with a large proportion of patients experiencing exacerbations, comorbidities, limitations in work, difficulties in social and family activities, and psychological distress. ${ }^{8}$

Bronchodilators are the cornerstone of COPD treatment. ${ }^{2}$ Long-acting $\beta_{2}$-agonists (LABAs), such as indacaterol, and long-acting muscarinic antagonists (LAMAs), such as tiotropium, are recommended as first choice or alternative treatments, either as monotherapies or in combination, for patients with all severities of COPD. ${ }^{2}$ Indacaterol maleate (Onbrez ${ }^{\circledR}$ Breezhaler $^{\circledR}$, Novartis, Basel, Switzerland) is a once-daily LABA, 
delivered by a low-resistance dry-powder inhaler, indicated for the maintenance treatment of airflow obstruction in adult COPD patients. ${ }^{9,10}$ In Phase III studies, indacaterol provided sustained 24-hour bronchodilation and significantly better efficacy in terms of lung function, symptom control, and health status compared with placebo, and comparable or superior efficacy compared with twice-daily LABAs (double blinded) and/or tiotropium (open label or blinded), ${ }^{11-15}$ and was well tolerated. ${ }^{16,17}$

Although very important, efficacy and safety data from clinical trials may not accurately reflect outcomes observed in routine clinical practice owing to their study designs and stringent inclusion/exclusion criteria. Noninterventional studies provide useful complementary information on realworld effectiveness and safety of treatments when prescribed in routine clinical practice and in particular circumstances, for example in different geographical regions and in ethnically diverse patient populations.

Despite the high COPD prevalence in the Middle East and North Africa, ${ }^{4}$ the BREATHE study reported that in these countries COPD is underdiagnosed and inadequately treated. ${ }^{18}$ The present study was designed to assess the effectiveness, treatment satisfaction, and safety of indacaterol and other long-acting inhaled bronchodilator therapies in COPD patients from the Middle East, Asia, and South Africa.

\section{Methods \\ Study design}

This was a 6-month, multicenter, prospective, noninterventional, real-world study, conducted between July 2011 and January 2014, to assess the effectiveness and safety of indacaterol and other inhaled long-acting bronchodilators in 12 countries in Asia, the Middle East, and South Africa. Therapy was prescribed according to the physician's judgment and clinical indication based on local prescribing information in the respective countries and was independent of the decision to include the patient in the study. Enrolled patients were observed over 6 months ( \pm 4 weeks) with recommended data-collection time points at baseline (study entry) and at months 1, 3, and 6/end of study. As the study was noninterventional, only data originating from routine clinical practice were collected, and there was no mandate for study-specific patient visits, tests, or monitoring. The study was conducted according to the declaration of Helsinki, the International Conference on Harmonisation Tripartite Guidelines for Good Clinical Practice, the Guidelines for Good Pharmacoepidemiology Practices of the International Society for Pharmacoepidemiology, and with applicable local regulations. The study protocol was approved by local ethics committees at participating centers. (Supplementary materials S1).

\section{Patients}

The study population consisted of male and female patients aged $\geq 40$ years, with mild-to-severe COPD (according to the Global initiative for chronic Obstructive Lung Disease [GOLD] 2009 strategy document), ${ }^{19}$ who were symptomatic, and were receiving indacaterol or other long-acting bronchodilators as monotherapy or in combination. Patients were current or ex-smokers with a smoking history of $\geq 10$ packyears. Eligible patients must have been either on 1) newly prescribed LABA or tiotropium as monotherapy or in combination, or 2) switched from one LABA to another or from a LABA to tiotropium (or vice versa). Patients gave their written informed consent for participation in the study.

Patients were not included if they had drug contraindications, a previous diagnosis of asthma, acute exacerbations (requiring antibiotics or hospitalizations) at study entry, unwillingness or inability to comply with the study requirements, treatment with inhaled corticosteroids (ICS) at study entry or within 3 months prior to study entry, or treatment with two different LABAs, LABA + ICS, tiotropium + ICS or triple therapy (tiotropium + LABA + ICS). However, new bronchodilator or ICS treatment could be initiated if required by the patient during the study.

\section{Objectives}

The objectives were to assess the effectiveness of treatments using the clinical COPD questionnaire (CCQ) to measure health status, to describe the characteristics of COPD patients treated with indacaterol and other long-acting bronchodilators, to evaluate patients' and physicians' satisfaction with treatment, to describe patients' and physicians' assessment of the inhaler used with indacaterol $\left(\right.$ Breezhaler $\left.^{\mathbb{R}}\right)$, to evaluate persistence with treatment, and to assess safety and tolerability of treatment.

\section{Assessments}

Health status was measured using the CCQ at baseline and end of study or at treatment change if the patient switched treatment (last observation carried forward). The CCQ was developed for use with COPD patients and comprises ten items divided into three domains: symptoms, functional state, and mental state. It generates an overall clinical COPD control score (as well as domain scores) between 0 (very good control) and 6 (extremely poor control). The questionnaire is short and easy to administer in the clinical practice setting, 
as required for a noninterventional study. The instrument has been shown to be valid, reliable, and responsive to change in COPD patients ${ }^{20,21}$ and has a minimal clinically important difference (MCID) of 0.4 points. ${ }^{22}$ The 7 -day recall version, validated in local languages, was used.

Persistence was defined as the percentage of patients receiving the treatment prescribed at baseline at each subsequent data collection time point until the end of study. Global satisfaction with treatment at end of study was recorded by patients on a four-point scale; physicians also rated treatment satisfaction based on assessment of effectiveness, tolerability, and compliance on a four-point scale. The inhaler used with indacaterol was assessed by patients as the percentage of patients reporting it as being very difficult, difficult, easy, or very easy to use, and by physicians in terms of the percentage of patients who clearly understood the use of the inhaler, used the device correctly but required further explanation, needed to have a number of technical points reviewed, and needed to have the use of the device explained again.

Safety and tolerability of treatment were assessed by patient- and physician-reported adverse events and serious adverse events, and the percentage of patients with adverse events and serious adverse events was determined.

Data were collected using paper case report forms designed for the study.

\section{Statistical analyses}

Data from studies across 12 countries (Bahrain, Egypt, India, Indonesia, Kuwait, Lebanon, Oman, Philippines, South Africa, Taiwan, Thailand, and the United Arab Emirates), conducted under one umbrella protocol, were pooled. The sample size for each country was based on the feasible numbers of patients treated with the respective drugs, rather than on statistical considerations; a ratio of 2:1 (indacaterol:other long-acting bronchodilators) was planned. The sample size calculation was set to test the primary endpoint, CCQ (end of study vs baseline in each arm; within-treatment comparisons) with a standard deviation estimated at 1.0 point. ${ }^{23}$ The precision was expressed in terms of the width of the $95 \%$ confidence interval.

The estimated CCQ total score precision was calculated for the respective sample sizes of each country. Changes from baseline in the CCQ total and domain scores were analyzed by a two-sided $t$-test; a $P$-value $<0.05$ indicates a significant difference from baseline. If no CCQ value was available at end of study, then the last postbaseline observation was carried forward. Differences between treatments were not analyzed.

Two populations were defined for analysis. The full analysis set comprised all patients who started treatment. The per-protocol population comprised the full analysis set, but excluded patients who deviated from the protocol-specified criteria of age $\geq 40$ years or baseline forced expiratory volume in 1 second $\left(\mathrm{FEV}_{1}\right)$ /forced vital capacity $(\mathrm{FVC})<70 \%$. The CCQ analysis was performed on the per-protocol population. Where ICS treatment was initiated during the study, additional CCQ assessments were carried out at the time of medication change; the study did not evaluate effectiveness postinitiation of ICS for these patients. Thus the effectiveness analysis is based on the population without ICS. All other analyses are based on the full analysis set, including patients treated with ICS during the study.

\section{Results}

Data were analyzed from 1,710 patients from 12 countries (Table 1). Indacaterol was most commonly used in the

Table I Participating patients (n, \%) by country and treatment

\begin{tabular}{|c|c|c|c|c|c|c|}
\hline & $\begin{array}{l}\text { Indacaterol } \\
(n=1,179)\end{array}$ & $\begin{array}{l}\text { Indacaterol + tiotropium } \\
(n=167)\end{array}$ & $\begin{array}{l}\text { Other LABA } \\
(n=68)\end{array}$ & $\begin{array}{l}\text { Tiotropium } \\
(\mathbf{n}=\mathbf{2 7} I)\end{array}$ & $\begin{array}{l}\text { Other LABA + tiotropium } \\
(n=25)\end{array}$ & $\begin{array}{l}\text { Total } \\
(\mathrm{N}=1,7 \mid 0)\end{array}$ \\
\hline Egypt & $103(8.7)$ & $32(19.2)$ & $7(10.3)$ & $14(5.2)$ & $22(88.0)$ & $178(10.4)$ \\
\hline India & $34(2.9)$ & 88 (52.7) & 0 & 0 & 0 & $122(7.1)$ \\
\hline Indonesia & $22(1.9)$ & 0 & 0 & $12(4.4)$ & 0 & $34(2.0)$ \\
\hline Middle East & $844(71.6)$ & $43(25.7)$ & 61 (89.7) & I84 (67.9) & $3(12.0)$ & I, I35 (66.4) \\
\hline Lebanon & $678(57.5)$ & $25(15.0)$ & $54(79.4)$ & $139(51.3)$ & $3(12.0)$ & $899(52.6)$ \\
\hline Bahrain & $20(1.7)$ & II (6.6) & 0 & $3(1.1)$ & 0 & $34(2.0)$ \\
\hline United Arab Emirates & $114(9.7)$ & 0 & I (I.5) & $38(14.0)$ & 0 & $153(8.9)$ \\
\hline Oman & $22(1.9)$ & 0 & $6(8.8)$ & $2(0.7)$ & 0 & $30(1.8)$ \\
\hline Kuwait & $10(0.8)$ & $7(4.2)$ & 0 & $2(0.7)$ & 0 & $19(1.1)$ \\
\hline Philippines & $40(3.4)$ & 0 & 0 & $9(3.3)$ & 0 & $49(2.9)$ \\
\hline Taiwan & $70(5.9)$ & $3(1.8)$ & 0 & $24(8.9)$ & 0 & $97(5.7)$ \\
\hline Thailand & $61(5.2)$ & 0 & 0 & $26(9.6)$ & 0 & $87(5.1)$ \\
\hline South Africa & $5(0.4)$ & I $(0.6)$ & 0 & $2(0.7)$ & 0 & $8(0.5)$ \\
\hline
\end{tabular}

Abbreviation: $L A B A$, long-acting $\beta_{2}$-agonist. 
Middle East/Lebanon, while indacaterol + tiotropium was most commonly used in India. Most of the "other LABA" use (generally formoterol) occurred in the Middle East/Lebanon, as was the case for tiotropium. The majority of the use of "other LABA + tiotropium" (mostly concurrent formoterol and tiotropium) occurred in Egypt.

Of the 1,710 patients analyzed, 1,535 (90\%) completed the study. Most discontinuations ( $n=129 / 175 ; 74 \%)$ were due to patients being lost to follow-up, other reasons included withdrawal of consent, adverse events, unsatisfactory therapeutic effect, death, protocol deviations, the condition no longer requiring the study drug, administrative problems, or missing data.

Patient demographics and baseline characteristics are shown in Table 2. Spirometry was available for approximately half of the total patients, as the preexisting diagnosis of COPD (according to GOLD 2009) did not have to be confirmed before study enrollment. Most patients $(n=1,337$; $78 \%$ ) had GOLD Stage II airflow limitation, as reported by the study physician, and the mean rate of exacerbations in the year prior to enrollment was 1.8. The two concurrent bronchodilator treatment groups contained the highest proportions of patients with severe/very severe airflow limitation ( $46 \%$ and $24 \%$ vs $12 \%-15 \%$ in other treatment groups) and had the worst overall CCQ scores at baseline (mean 2.7 and 3.3 vs $2.2-2.4$ in other treatment groups).

Overall, 40\% ( $n=677 / 1,710)$ of patients had switched treatment at baseline, prior to study entry, generally for reasons of insufficient disease control (77\% of switched patients; $n=520 / 677$ ).

\section{Efficacy}

The overall CCQ score improved significantly from baseline in all treatment groups (all $P<0.0001$; Figure 1). In each case, the level of improvement exceeded the MCID of 0.4 points. A similar pattern of significant improvements was observed for scores in the individual domains (symptoms, functional state, and mental state) in each treatment group (Figure S2).

Persistence with the assigned treatment to end of study was $>80 \%$ for all treatments except for the indacaterol + tiotropium combination, for which $66 \%$ of patients were persistent. Overall, 78\% of patients were satisfied/very satisfied with their current treatment (Figure 2). In terms of physicians' opinions (Figure 3), the effectiveness of treatment was rated good/very good for $76 \%$ of patients, the tolerability of treatment was rated good/very good for $79 \%$, and the adherence to treatment was judged good/very good in $78 \%$ of patients.
The inhaler used with indacaterol was rated as easy/very easy to use by the majority of patients receiving indacaterol either as monotherapy $(n=1,008 ; 85 \%)$ or concurrently with tiotropium ( $\mathrm{n}=113 ; 68 \%$ ) (Figure 4). Most physicians considered the use of this device to be clearly understood by their patients when receiving indacaterol as monotherapy $(n=918 ; 78 \%)$ or concurrently with tiotropium $(n=105 ; 63 \%)$ (Figure 5).

\section{Safety}

Adverse events were reported by $15 \%$ of all patients, ranging from $10 \%$ to $36 \%$ across the treatments. Cough was the most commonly reported adverse event $(n=68 ; 4 \%$ of total patients; Table 3). Serious adverse events were reported by $1.5 \%$ of all patients (Table 3 ). Five patients died during the study; four from the indacaterol treatment group (myocardial infarction, paracetamol overdose, prostate cancer, and sudden death) and one from the tiotropium group (reason unknown). Ten patients discontinued treatment because of adverse/serious adverse events.

\section{Discussion}

Despite the high prevalence of COPD in this geographical region, little is known about disease management and treatment in these countries. Data from the BREATHE study suggest that as few as $29 \%$ of COPD patients in the Middle East and North Africa receive respiratory treatment and that only $54 \%$ of those receive $\beta_{2}$-agonists, anticholinergics, and/or ICS. ${ }^{18}$ This large, noninterventional study is the first to evaluate the effects of long-acting bronchodilators in real-life clinical practice in a diverse region of 12 countries, nearly all from the Middle East and Asia. Most patients (83\%) had mild or moderate airflow limitation (GOLD Stages I and II), although the frequency of exacerbations appears higher than that reported in clinical trials in patients with more severe disease, ${ }^{24,25}$ and this may be reflected in the moderately high baseline CCQ scores. ${ }^{26}$ The mean baseline scores would categorize the patients as having "more symptoms" according to the current GOLD model for patient risk assessment, which states an approximate CCQ cutoff value of 1.0-1.5 points. ${ }^{2}$

Indacaterol and other long-acting bronchodilators improved health status in these patients, as shown by reductions from baseline in the overall and individual CCQ scores, by a level greater than the MCID. They were also regarded as effective treatments by most patients and physicians. The change from baseline CCQ score was numerically greatest in the concurrent indacaterol/tiotropium treatment group compared with all other subgroups. Although the differences 


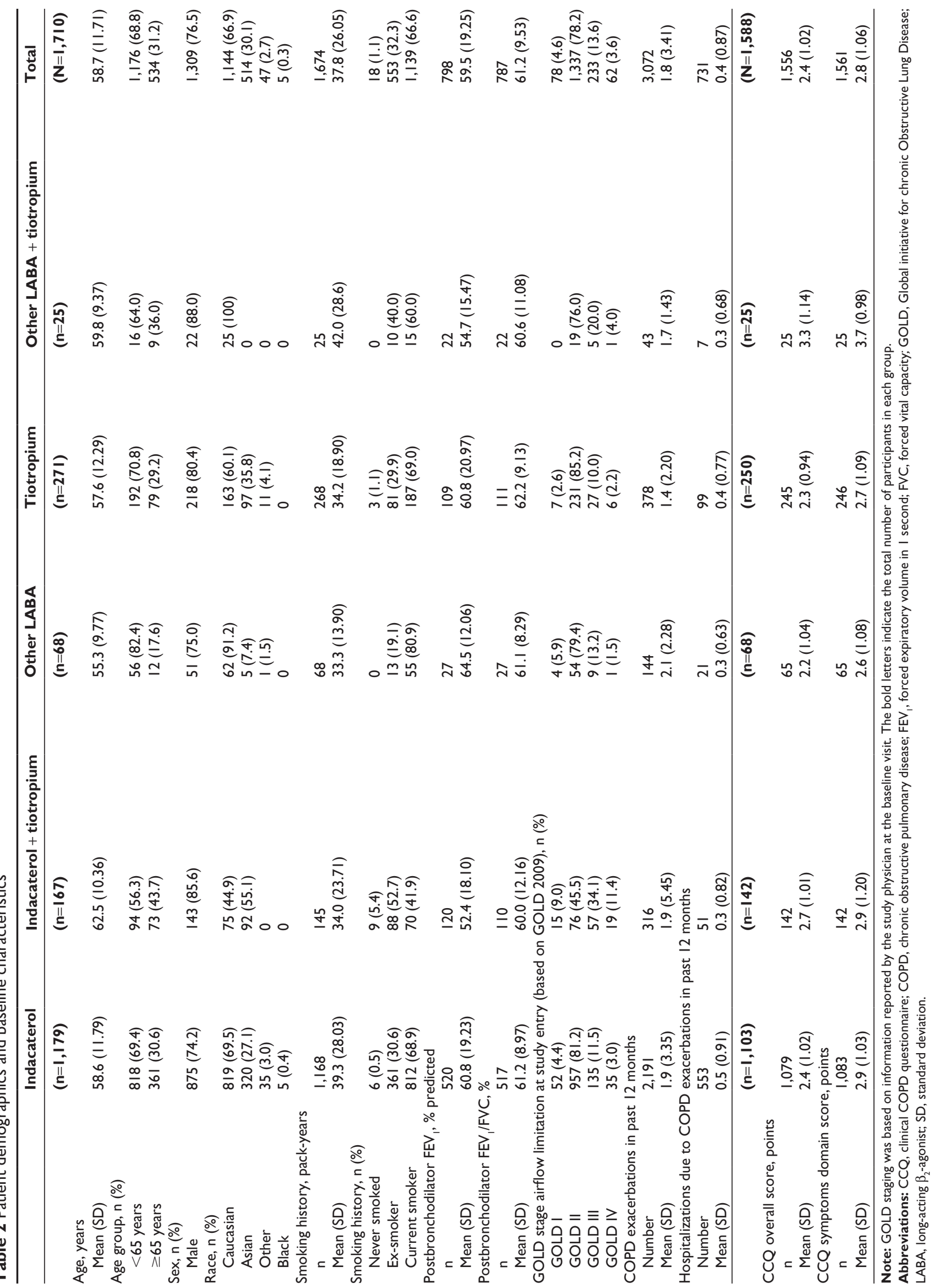




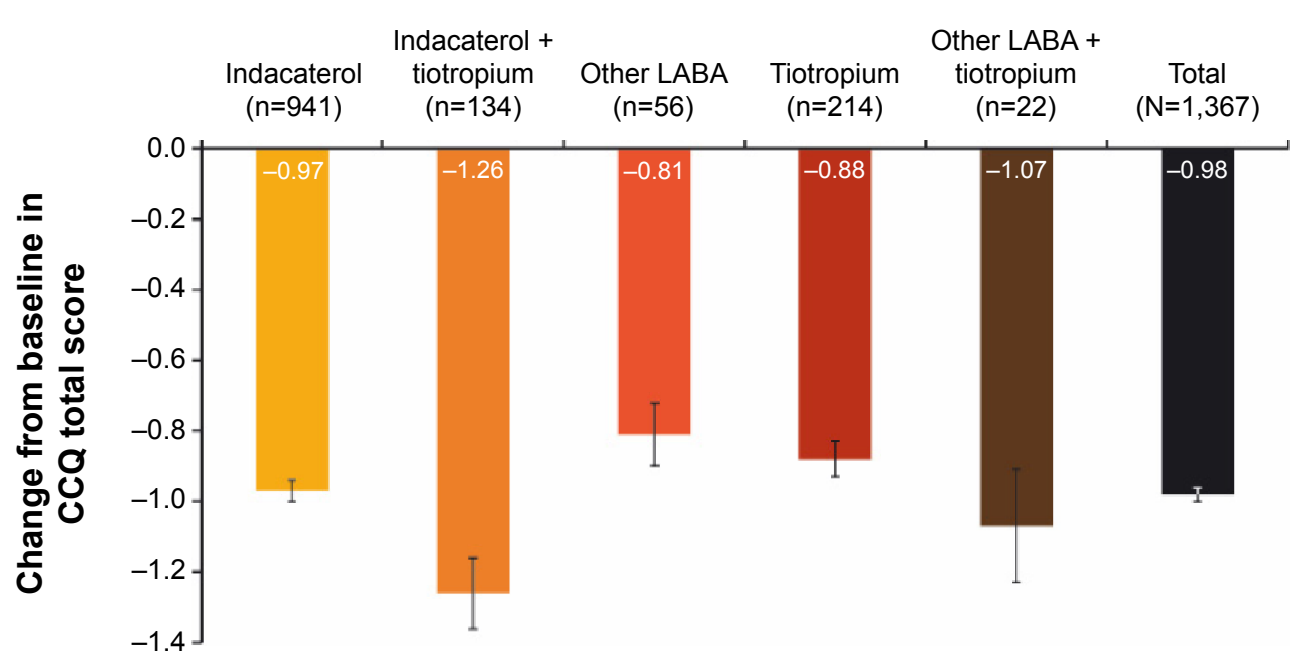

Figure I Changes from baseline in CCQ overall score.

Notes: Data are mean \pm standard error. Last observation was carried forward. All $P<0.000$ I for change in CCQ overall score from baseline to end of study. Abbreviations: CCQ, clinical COPD questionnaire; LABA, long-acting $\beta_{2}$-agonist.

between treatments were not subject to formal statistical analysis, and patient numbers in this group were small, this interesting observation seems to be validated by a recent study evaluating the efficacy of combination LABA/LAMA treatments. ${ }^{27}$ However, the concurrent indacaterol/tiotropium treatment in the present study also attracted the lowest ratings for patient and physician satisfaction compared with the other subgroups, for reasons that are unknown but may have involved the need to use two different inhalers.

The inhaler used with indacaterol was rated as easy/ very easy to use by $85 \%$ of patients using indacaterol as monotherapy and by $68 \%$ using it with concurrent tiotropium, perhaps again because patients found two inhalers harder to manage. Physicians also rated the inhaler highly in terms of how well patients understood its use. This was shown previously by Chapman et $\mathrm{al}^{28}$ who reported that $81 \%$ of patients used the indacaterol inhaler without a critical error on the first day of use, compared with $70 \%$ of patients when they used the single-dose, dry-powder inhaler for tiotropium. Additionally, in that study, significantly more patients chose the indacaterol inhaler as their preferred inhaler to use on a daily basis ( $61 \%$ of patients vs $31 \%$ for the tiotropium device) ${ }^{28}$

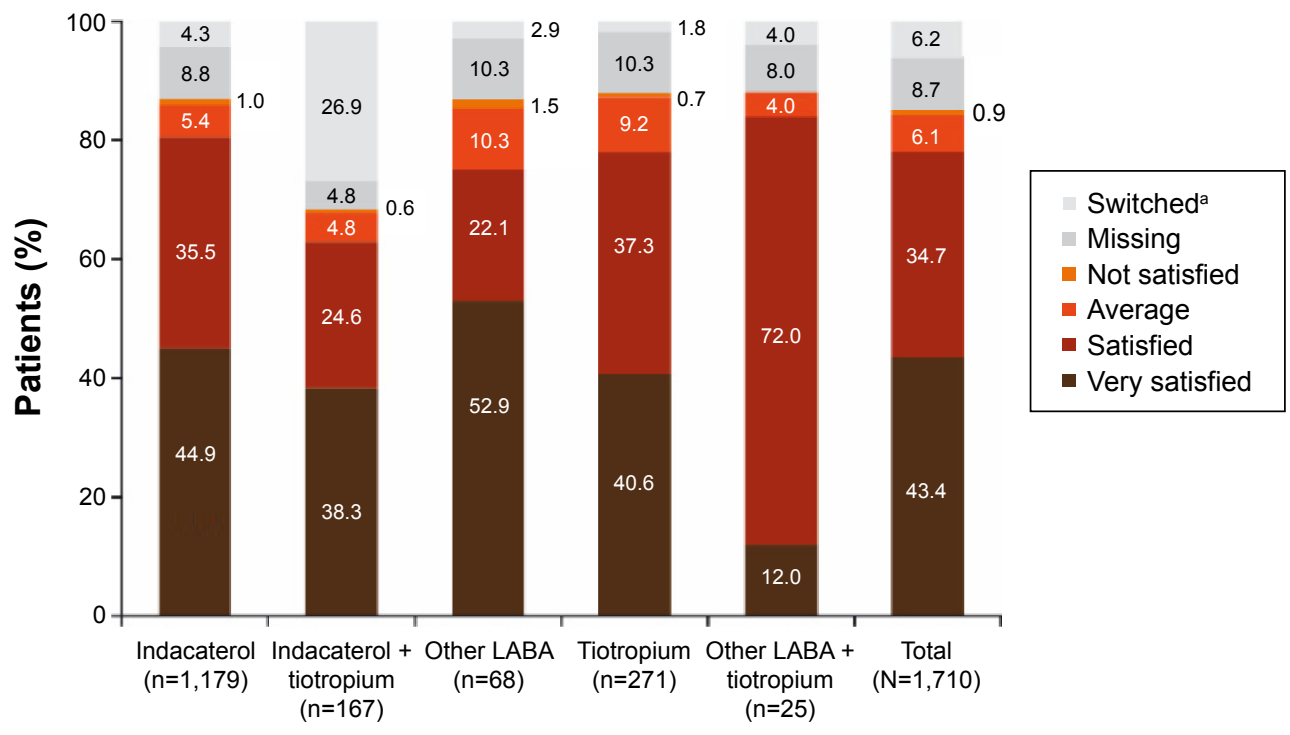

Figure 2 Patient satisfaction with current treatment at end of study.

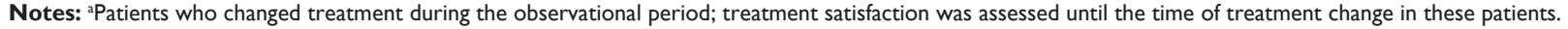
Abbreviation: LABA, long-acting $\beta_{2}$-agonist. 


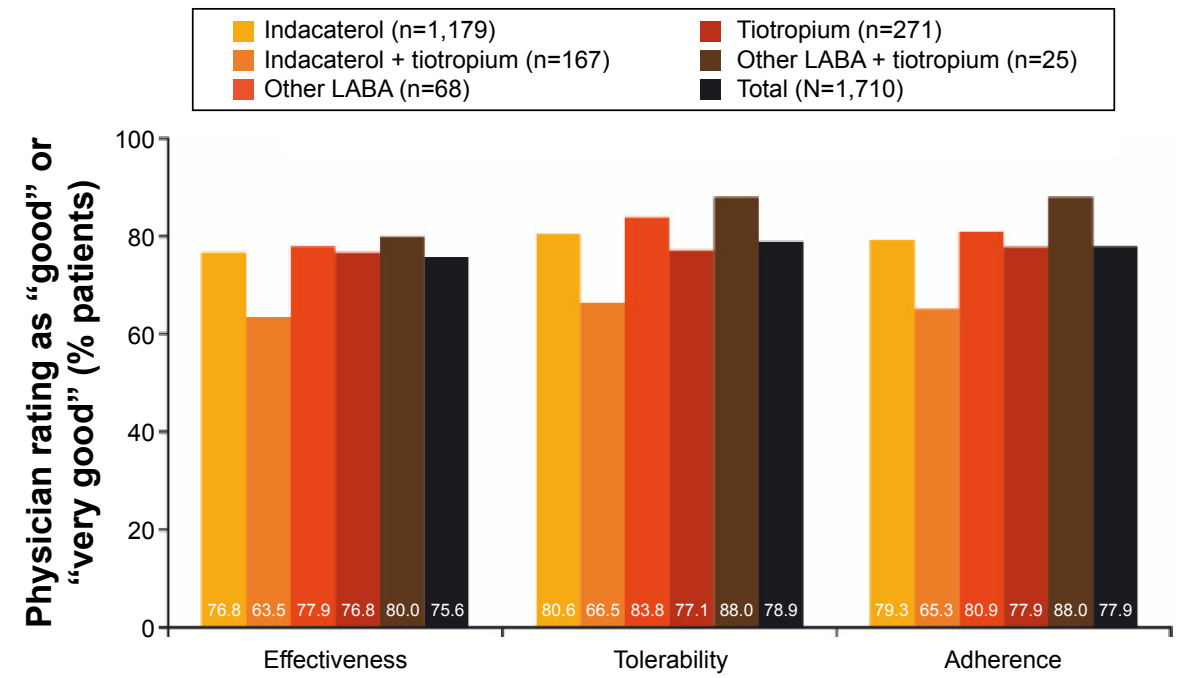

Figure 3 Physician satisfaction with current treatment at end of study.

Note: Patients who changed treatment cohort during the observational period were not included in the analysis.

Abbreviation: LABA, long-acting $\beta_{2}$-agonist.

In a study of patients' and physicians' preferences for inhaler devices, ease-of-use was reported by $66 \%$ of patients as the most important attribute for an inhaler. ${ }^{29}$

Indacaterol and other long-acting bronchodilators had acceptable safety and tolerability profiles. The only possible exception was in the "other LABA + tiotropium" group, where the frequency of adverse events was notably higher, but the number of patients was small, and so it may be a random finding. While adverse event reporting procedures were standardized across the different participating countries, the difference may reflect national variations (most of the patients in this treatment group were from Egypt) rather than any real safety differences. The treatments evaluated in this study have all previously been shown in clinical trials to have acceptable safety and tolerability profiles that were comparable with placebo data. ${ }^{17}$

Randomized clinical trials provide most of the scientific evidence to guide treatment recommendations and decisions. However, one study suggests that patients included in COPD clinical trials may represent only $17 \%$ of the real-life COPD patient population, ${ }^{30}$ which makes it difficult to extrapolate clinical trial data to real-life prescribing and outcomes. Reallife studies have an increasingly valuable role in respiratory medicine, to complement the results from clinical studies. ${ }^{31}$

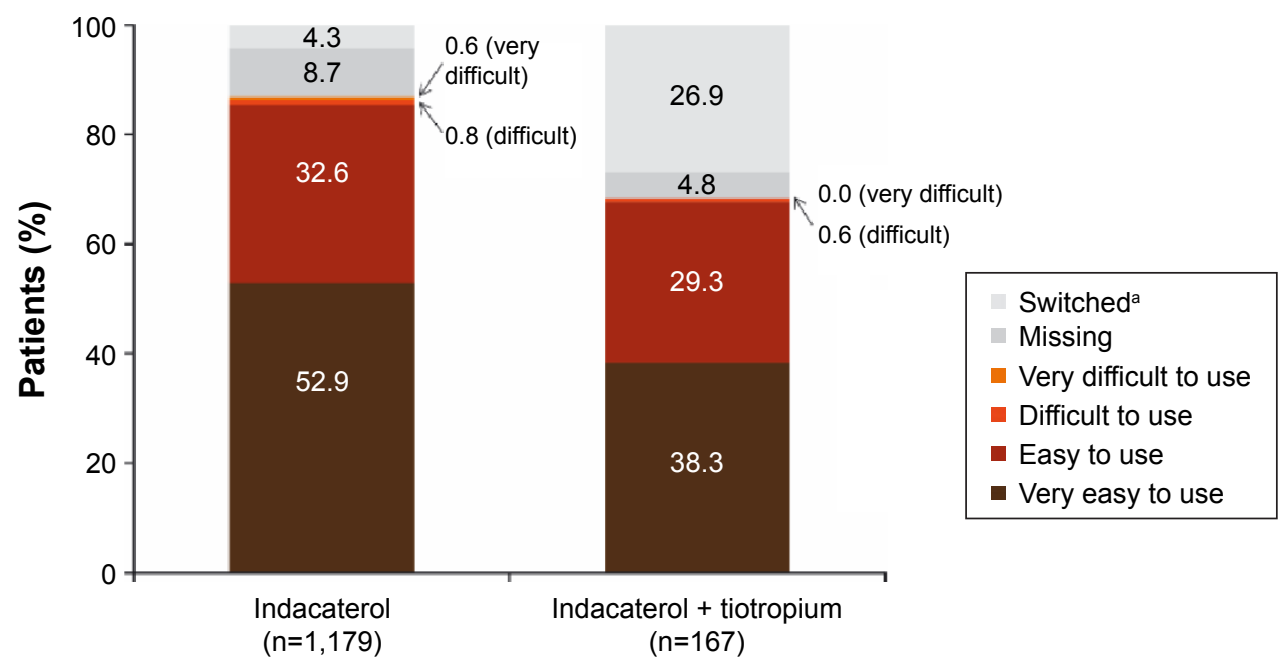

Figure 4 Patient assessment of ease of use of indacaterol inhaler at end of study.

Notes: aPatients who changed treatment during the observational period; inhaler ease of use was assessed until the time of treatment change in these patients. 


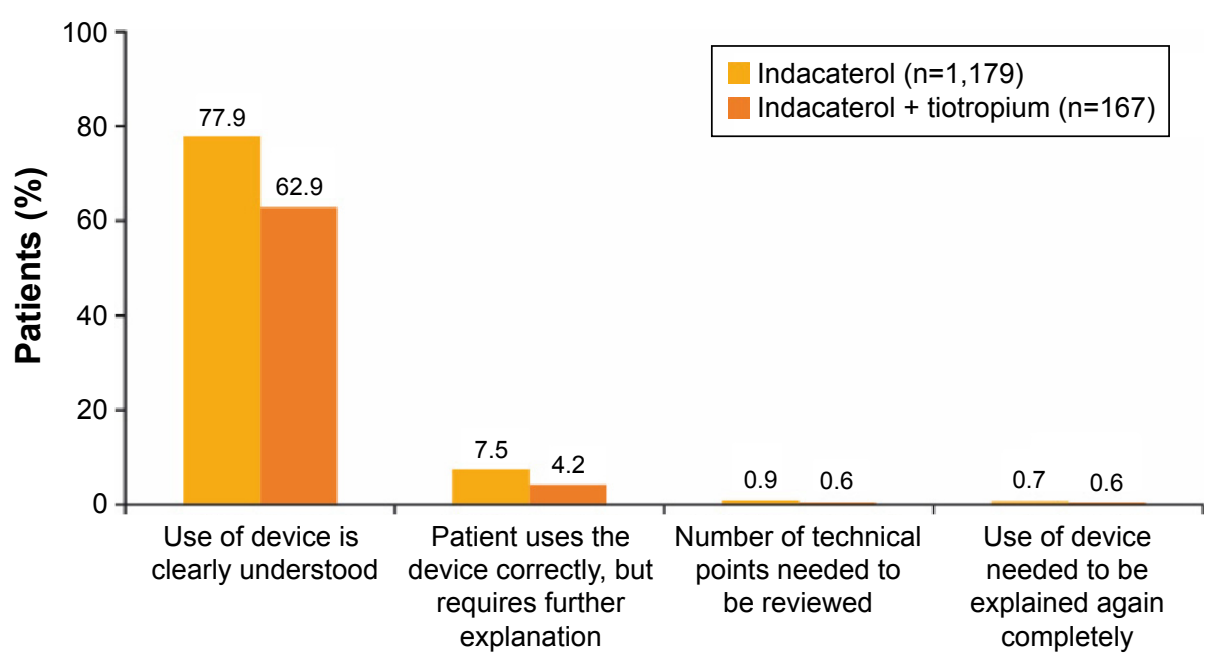

Figure 5 Physician assessment of indacaterol inhaler ease of use at end of study.

Note: Patients who changed treatment cohort during the observational period were not included in the analysis.

To date, several small observational studies of indacaterol treatment in COPD patients have been conducted and have reported results broadly consistent with our findings. A study of 28 outpatients in Japan showed indacaterol to be effective and well tolerated; all participants found the indacaterol inhaler easy to use. ${ }^{32}$ In the UK, a small cohort of patients who were not well controlled on current therapy (ICS-based in 8 of 15 patients) fared better in terms of exacerbations and health status after switching to indacaterol alone or in combination with tiotropium. ${ }^{33}$ Another small study in 23 Japanese patients found improved physical activity in patients who received indacaterol for 4 weeks. ${ }^{34}$ In comparison with these studies, our own study is notable both for its size and for the diverse region covered.

Although the study included patients from 12 countries, the majority were from the Middle East, notably Lebanon, and Egypt. Our results will therefore be more specific to these two countries rather than to all countries involved with the study. In future studies, it may be more appropriate to stratify enrollment by country according to the population size and COPD prevalence in each country.

It should be noted that the intended ratio of $2: 1$ for patients on indacaterol:other long-acting bronchodilators was not achieved in this real-life study. However, as the study was

Table 3 Patients (n, \%) with AEs and SAEs

\begin{tabular}{|c|c|c|c|c|c|c|}
\hline & Indacaterol $(n=I, \mid 79)$ & $\begin{array}{l}\text { Indacaterol + } \\
\text { tiotropium }(n=167)\end{array}$ & $\begin{array}{l}\text { Other LABA } \\
(n=68)\end{array}$ & $\begin{array}{l}\text { Tiotropium } \\
(n=27 I)\end{array}$ & $\begin{array}{l}\text { Other LABA + } \\
\text { tiotropium }(n=25)\end{array}$ & $\begin{array}{l}\text { Total } \\
(\mathbf{N}=I, 7 \mid 0)\end{array}$ \\
\hline Total AEs, n (\%) & $183(15.5)$ & $16(9.6)$ & $10(14.7)$ & $43(15.9)$ & $9(36.0)$ & $261(15.3)$ \\
\hline \multicolumn{7}{|c|}{ Number (\%) of patients with most frequent AEs (occurring in $\geq 3.0 \%$ of patients in any group) } \\
\hline Cough & $50(4.2)$ & $7(4.2)$ & $3(4.4)$ & $8(3.0)$ & 0 & $68(4.0)$ \\
\hline COPD worsening & $17(1.4)$ & $\mathrm{I}(0.6)$ & $2(2.9)$ & $3(1.1)$ & $4(16.0)$ & $27(1.6)$ \\
\hline Productive cough & $7(0.6)$ & 0 & 0 & $2(0.7)$ & I (4.0) & $10(0.6)$ \\
\hline Dyspepsia & $3(0.3)$ & 0 & $\mathrm{I}(\mathrm{I} .5)$ & $\mathrm{I}(0.4)$ & $2(8.0)$ & $7(0.4)$ \\
\hline Headache & $5(0.4)$ & 0 & 0 & $\mathrm{I}(0.4)$ & I (4.0) & $7(0.4)$ \\
\hline LRTI & $2(0.2)$ & $2(1.2)$ & 0 & $\mathrm{I}(0.4)$ & I (4.0) & $6(0.4)$ \\
\hline Infection & $4(0.3)$ & 0 & 0 & 0 & I (4.0) & $5(0.3)$ \\
\hline Chest pain & $2(0.2)$ & 0 & $\mathrm{I}(\mathrm{I} .5)$ & 0 & I (4.0) & $4(0.2)$ \\
\hline Tonsillitis & $\mathrm{I}(0.1)$ & 0 & 0 & 0 & $2(8.0)$ & $3(0.2)$ \\
\hline Diarrhea & $\mathrm{I}(0.1)$ & 0 & 0 & 0 & I (4.0) & $2(0.1)$ \\
\hline $\begin{array}{l}\text { Discontinuation due to } \\
\text { AE/SAE, } n(\%)\end{array}$ & $5(0.4)$ & 0 & 0 & $5(1.8)$ & 0 & $10(0.6)$ \\
\hline SAEs, n (\%) & $15(1.3)$ & $4(2.4)$ & 0 & $7(2.6)$ & 0 & $26(1.5)$ \\
\hline Deaths, n (\%) & $4(0.3)$ & 0 & 0 & I (0.4) & 0 & $5(0.3)$ \\
\hline
\end{tabular}

Abbreviations: AE, adverse event; COPD, chronic obstructive pulmonary disease; LABA, long-acting $\beta_{2}$-agonist; LRTI, lower respiratory tract infection; SAE, serious adverse event. 
not designed for between-group comparisons but for withintreatment comparisons (end of study vs baseline), we did not feel that this imbalance would influence the study results.

A small number of never-smokers ( $1 \%$ of total) were enrolled in the study despite the requirement for patients to have a minimum smoking history. These patients were included because they had been diagnosed by the treating physicians as having COPD. While exposure to tobacco smoke is a strong risk factor for COPD, exposure to smoke from home cooking and indoor fuels, and to occupational dust and chemicals and air pollution, are also important contributory factors to the disease..$^{2,5}$

This study was designed and conducted when COPD severity was determined solely by airflow limitation, according to the 2009 version of GOLD. Since 2011, GOLD has recommended that patients are assessed in terms of exacerbation risk according to airflow limitation, symptom severity, and history of exacerbations. ${ }^{2}$ Some of the patients in the present study with more severe airflow limitation may have approximated to the current higher-risk GOLD groups, for which the GOLD-recommended first choice treatments are $\mathrm{ICS}+\mathrm{LABA}+\mathrm{LAMA}$, ICS + LABA, or LAMA. ${ }^{2}$ Treatments in this study were given according to local prescribing information and at the discretion of the treating physician, independently of the inclusion of the patients in the study. Patients treated with ICS at study entry, or within 3 months prior to study entry, were not eligible for inclusion; however, ICS treatment could have been initiated during the study, if the treating physician felt that it was required. Furthermore, combinations of long-acting bronchodilators are listed as alternative choices in the current GOLD treatment recommendations, ${ }^{2}$ and baseline data show that these treatments were being given appropriately to patients with more severe disease, in terms of both airflow limitation and symptoms, compared with the other treatment groups.

It should also be noted that exacerbations were not recorded during the study period as they were not an endpoint in the study. This may be a limitation of the study as the possibility of an exacerbation coinciding with the patient's visit for assessment of CCQ scores, and therefore potentially impacting the CCQ scores, cannot be excluded.

In conclusion, the present results support previous findings from randomized controlled clinical trials demonstrating the efficacy and safety of indacaterol and build on these in a geographical region that has been sparsely studied thus far. In a real-world setting, indacaterol and other longacting bronchodilators provided a substantial and clinically important improvement in health status, and the indacaterol treatment and inhaler were well received by both patients and physicians.

\section{Acknowledgments}

The authors acknowledge Sashka Hristoskova (Novartis Pharma AG) for critical review of the manuscript. Lietta Nicolaides and Sarah Filcek (CircleScience, an Ashfield Company, part of UDG Healthcare plc) provided medical writing assistance. This was funded by Novartis, the INFLOW study sponsor.

\section{Disclosure}

GJ has received payments from AstraZeneca and Novartis (fees and honoraria for lecturing, advisory membership); MSD (fees and honoraria for lecturing, advisory membership, speakers' bureau); Abbott, Boehringer Ingelheim, and Takeda (fees and honoraria for lecturing). WE-S has received lecturing honoraria from AstraZeneca, GlaxoSmithKline, MSD, and Pfizer. CP has received consultancy and speaker fees from Novartis, and speaker fees from AstraZeneca and GlaxoSmithKline. FY has received speaker and advisory board fees from AstraZeneca, Bayer, Boehringer Ingelheim, GlaxoSmithKline, and Novartis; and speaker fees from Takeda and Zambon. TdeG has received advisory board fees from Novartis. H-PK has received speaker and advisory board fees from Novartis, and speaker fees from Bayer, Boehringer Ingelheim, Eli Lilly, GlaxoSmithKline, Pfizer, Roche, and Takeda. All investigators (GJ, WE-S, CP, FY, TdeG, and $\mathrm{H}-\mathrm{PK}$ ) received fees from Novartis for conducting the study. SBP and VP are Novartis employees. VP is also a Novartis shareholder. The authors report no other conflicts of interest in this work.

\section{References}

1. World Health Organization (WHO). Burden of COPD. Available from http://www.who.int/respiratory/copd/burden/en/. Accessed October 29 , 2014.

2. Global Initiative for Chronic Obstructive Lung Disease (GOLD 2014). Global Strategy for the Diagnosis, Management, and Prevention of Chronic Obstructive Pulmonary Disease (Updated 2014). Available from: http://www.goldcopd.org/uploads/users/files/GOLD_Report_2014.pdf. Accessed November 13, 2014.

3. Buist AS, McBurnie MA, Vollmer WM, et al. International variation in the prevalence of COPD (the BOLD Study): a population-based prevalence study. Lancet. 2007;370(9589):741-750.

4. Tageldin MA, Nafti S, Khan JA, et al. Distribution of COPD-related symptoms in the Middle East and North Africa: results of the BREATHE study. Respir Med. 2012;106(Suppl 2):S25-S32.

5. Regional COPD Working Group. COPD prevalence in 12 Asia-Pacific countries and regions: projections based on the COPD prevalence estimation model. Respirology. 2003;8(2):192-198.

6. Idolor LF, DE Guia TS, Francisco NA, et al. Burden of obstructive lung disease in a rural setting in the Philippines. Respirology. 2011;16(7): $1111-1118$ 
7. McKay AJ, Mahesh PA, Fordham JZ, Majeed A. Prevalence of COPD in India: a systematic review. Prim Care Respir J. 2012;21(3):313-321.

8. Uzaslan E, Mahboub B, Beji M, et al. The burden of chronic obstructive pulmonary disease in the Middle East and North Africa: results of the BREATHE study. Respir Med. 2012;106(Suppl 2):S45-S59.

9. Onbrez Breezhaler ${ }^{\mathbb{B}} 150$ and 300 microgram inhalation powder, hard capsules [UK Summary of Product Characteristics]. Updated October 23, 2014. Surrey, UK: Novartis Pharmaceuticals UK Ltd. Available from: http://www.medicines.org.uk/EMC/medicine/23260/SPC/Onb rez+Breezhaler+150+and $+300+$ microgram+inhalation+powder $\% 2 \mathrm{c}+$ hard+capsules/. Accessed October 29, 2014.

10. Pavkov R, Mueller S, Fiebich K, et al. Characteristics of a capsule based dry powder inhaler for the delivery of indacaterol. Curr Med Res Opin. 2010;26(11):2527-2533.

11. Kornmann O, Dahl R, Centanni S, et al. Once-daily indacaterol versus twice-daily salmeterol for COPD: a placebo-controlled comparison. Eur Respir J. 2011;37(2):273-279.

12. Korn S, Kerwin E, Atis S, et al. Indacaterol once-daily provides superior efficacy to salmeterol twice-daily in COPD: a 12-week study. Respir Med. 2011;105(5):719-726.

13. Donohue JF, Fogarty C, Lötvall J, et al. Once-daily bronchodilators for chronic obstructive pulmonary disease: indacaterol versus tiotropium. Am J Respir Crit Care Med. 2010;182(2):155-162.

14. Dahl R, Chung KF, Buhl R, et al. Efficacy of a new once-daily longacting inhaled $\beta_{2}$-agonist indacaterol versus twice-daily formoterol in COPD. Thorax. 2010;65(6):473-479.

15. Buhl R, Dunn LJ, Disdier C, et al. Blinded 12-week comparison of oncedaily indacaterol and tiotropium in COPD. Eur Respir J. 2011;38(4): 797-803.

16. Chapman KR, Rennard SI, Dogra A, et al. Long-term safety and efficacy of indacaterol, a long-acting $\beta_{2}$-agonist, in subjects with COPD: a randomized, placebo-controlled study. Chest. 2011;140(1):68-75.

17. Donohue JF, Singh D, Kornmann O, Lawrence D, Lassen C, Kramer B. Safety of indacaterol in the treatment of patients with COPD. Int $J$ Chron Obstruct Pulmon Dis. 2011;6:477-492.

18. Idrees M, Koniski ML, Taright $\mathrm{S}$, et al. Management of chronic obstructive pulmonary disease in the Middle East and North Africa: results of the BREATHE study. Respir Med. 2012;106(Suppl 2):S33-S44.

19. Global Initiative for Chronic Obstructive Lung Disease (GOLD 2009). Global Strategy for the Diagnosis, Management, and Prevention of Chronic Obstructive Pulmonary Disease (Updated 2009). Available from: www.goldcopd.com. Accessed October 29, 2014.

20. Kocks JW, Kerstjens HA, Snijders SL, et al. Health status in routine clinical practice: validity of the clinical COPD questionnaire at the individual patient level. Health Qual Life Outcomes. 2010;8:135.

21. van der Molen T, Willemse BW, Schokker S, ten Hacken NH, Postma DS, Juniper EF. Development, validity and responsiveness of the Clinical COPD Questionnaire. Health Qual Life Outcomes. 2003;1:13.
22. Kocks JW, Tuinenga MG, Uil SM, van den Berg JW, Ståhl E, van der Molen T. Health status measurement in COPD: the minimal clinically important difference of the clinical COPD questionnaire. Respir Res. 2006;7:62.

23. Ställberg B, Nokela M, Ehrs PO, Hjemdal P, Jonsson EW. Validation of the clinical COPD Questionnaire (CCQ) in primary care. Health Qual Life Outcomes. 2009;7:26.

24. Calverley PM, Anderson JA, Celli B, et al. Salmeterol and fluticasone propionate and survival in chronic obstructive pulmonary disease. N Engl J Med. 2007;356(8):775-789.

25. Wedzicha JA, Calverley PM, Seemungal TA, et al. The prevention of chronic obstructive pulmonary disease exacerbations by salmeterol/ fluticasone propionate or tiotropium bromide. Am J Respir Crit Care Med. 2008;177(1):19-26.

26. Kocks JW, van den Berg JW, Kerstjens HA, et al. Day-to-day measurement of patient-reported outcomes in exacerbations of chronic obstructive pulmonary disease. Int J Chron Obstruct Pulmon Dis. 2013;8: 273-286.

27. Bateman ED, Ferguson GT, Barnes N, et al. Dual bronchodilation with QVA149 versus single bronchodilator therapy: the SHINE study. Eur Respir J. 2013;42(6):1484-1494.

28. Chapman KR, Fogarty CM, Peckitt C, et al. Delivery characteristics and patients' handling of two single-dose dry-powder inhalers used in COPD. Int J Chron Obstruct Pulmon Dis. 2011;6:353-363.

29. Molimard M, Colthorpe P. Inhaler devices for chronic obstructive pulmonary disease: insights from patients and healthcare practitioners. J Aerosol Med Pulm Drug Deliv. 2015;28(3):219-228.

30. Herland K, Akselsen JP, Skjønsberg OH, Bjermer L. How representative are clinical study patients with asthma or COPD for a larger "real life" population of patients with obstructive lung disease? Respir Med. 2005;99(1):11-19.

31. Saturni S, Bellini F, Braido F, et al. Randomized controlled trials and real life studies. Approaches and methodologies: a clinical point of view. Pulm Pharmacol Ther. 2014;27(2):129-138.

32. Ohno T, Wada S, Hanada S, Sawaguchi H, Muraki M, Tohda Y. Efficacy of indacaterol on quality of life and pulmonary function in patients with COPD and inhaler device preferences. Int J Chron Obstruct Pulmon Dis. 2014;9:107-114.

33. Singh MP. Indacaterol therapy in moderate-to-severe chronic obstructive pulmonary disease: findings from a single-center primary care cohort. Int J Chron Obstruct Pulmon Dis. 2013;8:613-619.

34. Hataji O, Naito M, Ito K, Watanabe F, Gabazza EC, Taguchi O. Indacaterol improves daily physical activity in patients with chronic obstructive pulmonary disease. Int J Chron Obstruct Pulmon Dis. 2013;8: $1-5$. 


\section{Supplementary materials}

\section{Supplementary S I}

\section{Ethics Committees and Institutional Review Boards}

The following Ethics Committees and Institutional Review Boards approved the study protocol: MOHP-RHD-REC, Cairo, Egypt; Institutional Review Board of the Hammoud Hospital University Medical Center, Saida, Lebanon; Dubai Heath Authority Ethics Committee, Dubai, United Arab Emirates; Joint Committee for the Protection of Human Subjects in Research of the Health Sciences Center (HSC) \& Kuwait Institute for Medical Specialization (KIMS), Safat, Kuwait; American Mission Hospita, Manama, Kingdome of Bahrain; National Health Regulatory Authority, Manama, Kingdome of Bahrain; Sultanate of Oman, Ministry of Health - Division General of Planning, Muscat, Oman; The Ethic Committee of the Faculty of Medicine, University of Indonesia, Jawa Barat, Indonesia; CLINICOM "SUSHRUTA", Bangalore, India; Ethics Committee, Fortis Hospitals, Kolkata, West Bengal, India; Philippine Heart Center (PHC) Ethics Review Committee, Quezon City, Philippines; Mary Mediatrix Medical Center (MMMC), Batangas, Philippines; St. Luke's Institutional Ethics Review Committee, Quezon City, Philippines; UPM Research Ethics Board (UPMREB), Manila, Philippines; Office of the Khon Kaen University Ethics Committee for Human Research, Khon
Kaen, Thailand; Institutional Review Board, Faculty of Medicine, Chulalongkorn University, Bangkok, Thailand; Office of Research Administration, Faculty of Medicine, Thammasat University Hospital, Pathum Thani, Thailand; The Research Ethics Committee, Faculty of Medicine, Prince of Songkla University, Songkla, Thailand; Research Ethics Committee, Faculty of Medicine, Chiang Mai University, Chiang Mai, Thailand; Institutional Review Board, Royal Thai Army Medical Department, Bangkok, Thailand; Ethics Committee Institute for the Development of Human Research Protections (IHRP), Nonthaburi, Thailand; Research Ethics Committee, Department of Medical Science, Ministry of Public Health Chest Disease Institute, Nonthaburi, Thailand; Institutional Review Board Committee B, Changhua Christian Hospital, Changhua, Taiwan; Institutional Review Board, Chung Shan Medical University Hospital, Chung Shan, Taiwan; Institutional Review Board, Cheng Hsin General Hospital, Taipei, Taiwan; Chang Gung Memorial Foundation Institutional Review Board, Linkou, Taiwan; Institutional Review Board, Cathay General Hospital, Taipei, Taiwan; Institutional Review Board I, Tri-Service General Hospital, Taipei, Taiwan; Institutional Review Board, Taichung Veteran's General Hospital, Taichung, Taiwan; Institutional Review Board, China Medical University Hospital, Taichung, Taiwan; Institutional Review Board, Tungs' Taichung MetroHarbor Hospital, Taichung, Taiwan; Pharma-Ethics (Pty) Ltd, Centurion, South Africa.

\section{Supplementary Figure S2}

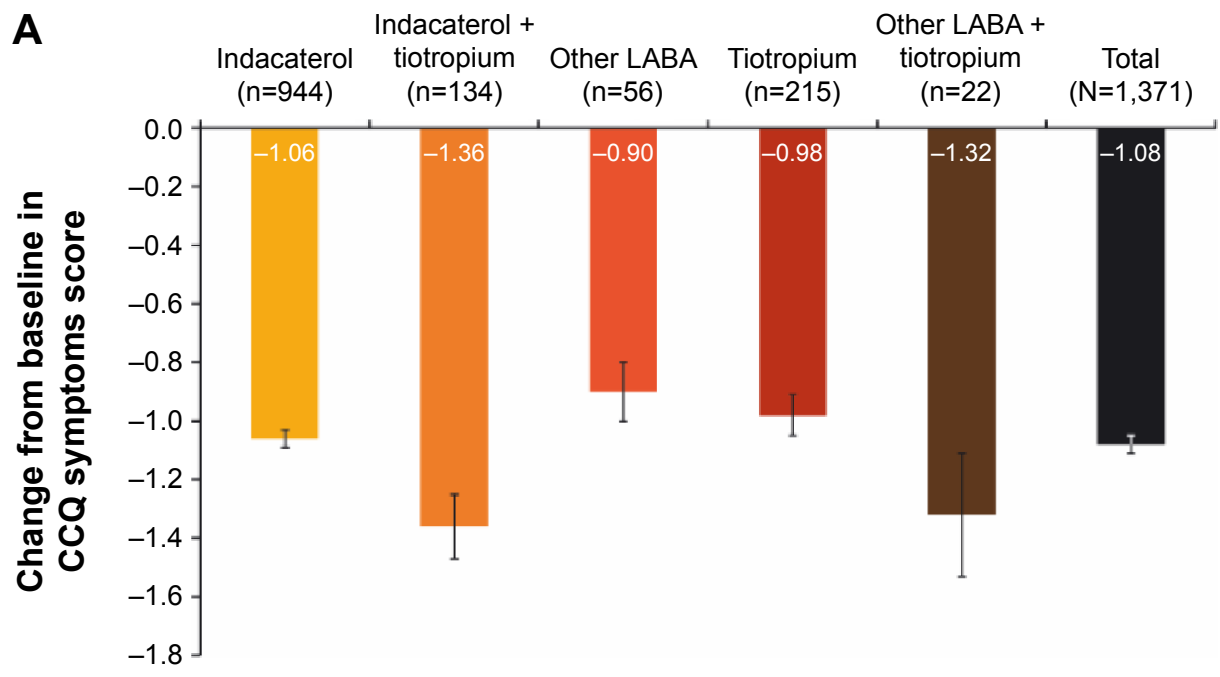

Figure S2 (Continued) 

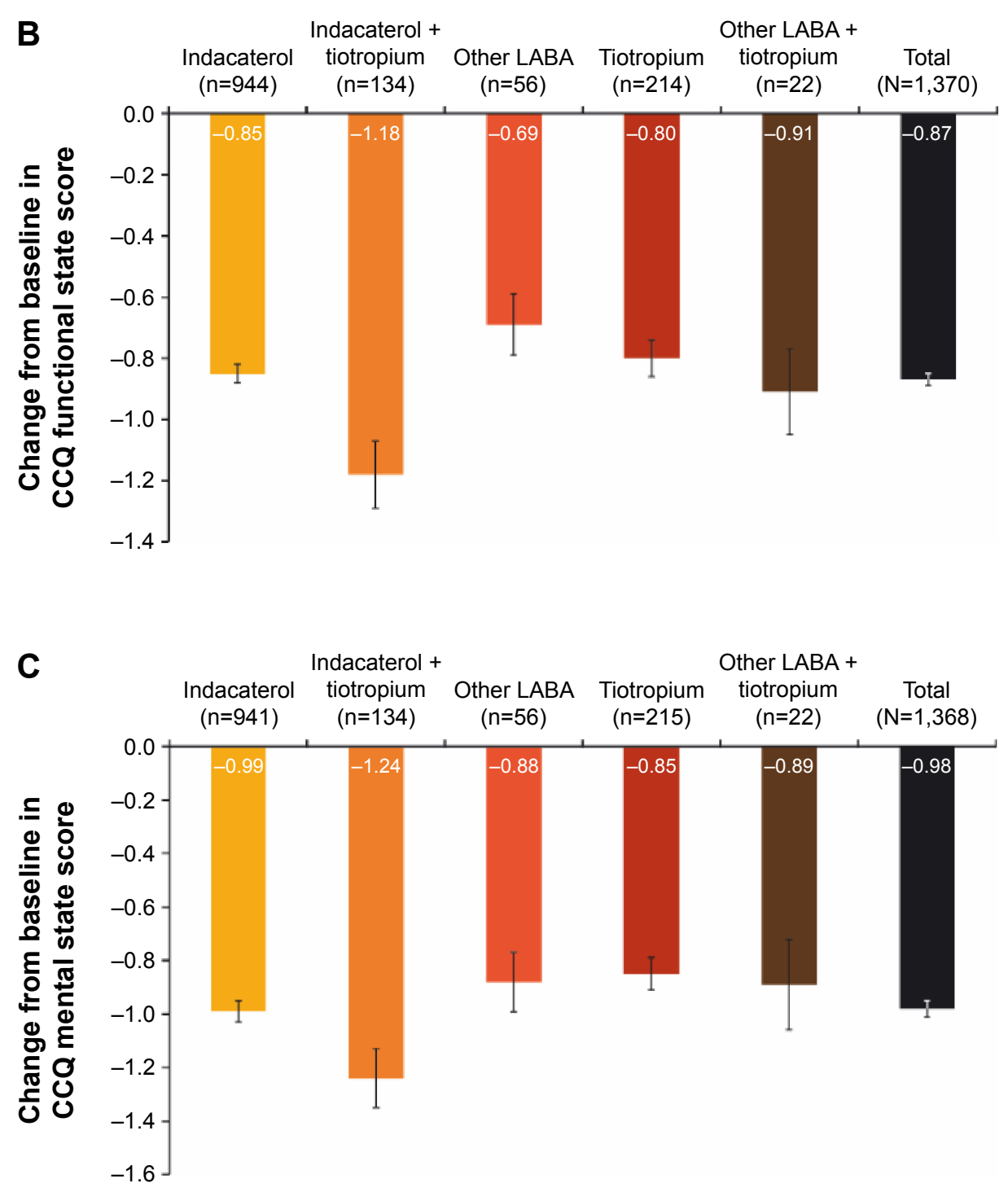

Figure S2 Change from baseline in CCQ (A) symptoms score, (B) functional state score and (C) mental state score.

Notes: Data are mean \pm standard error. Last observation was carried forward. All $P<0.000$ I for change in CCQ from baseline to end of study. Per-protocol population. Abbreviations: CCQ, clinical COPD questionnaire; LABA, long-acting $\beta_{2}$-agonist.

\section{Publish your work in this journal}

The International Journal of COPD is an international, peer-reviewed journal of therapeutics and pharmacology focusing on concise rapid reporting of clinical studies and reviews in COPD. Special focus is given to the pathophysiological processes underlying the disease, intervention programs, patient focused education, and self management protocols.

\section{Dovepress}

This journal is indexed on PubMed Central, MedLine and CAS. The manuscript management system is completely online and includes a very quick and fair peer-review system, which is all easy to use. Visit http://www.dovepress.com/testimonials.php to read real quotes from published authors. 